\title{
Field Evaluation of LED Fluorescence Microscopy for Demonstration of Trypanosoma brucei rhodesiense in Patient Blood
}

\author{
Enock Matovu1, Andrew Edielu², James 0jom², Ann Nanteza1, Charles Drago Kato', \\ Sylvain Biéler ${ }^{3 *}$, Joseph Mathu Ndung' $\mathbf{u}^{3}$ \\ ${ }^{1}$ College of Veterinary Medicine, Animal Resources and Biosecurity, Makerere University, Kampala, Uganda \\ ${ }^{2}$ Lwala Hospital, Soroti, Uganda \\ ${ }^{3}$ Foundation for Innovative New Diagnostics (FIND), Campus Biotech, Chemin des Mines 9, Geneva, Switzerland \\ Email:matovue04@yahoo.com, andrew.edielu@gmail.com, jamesojom@gmail.com,nantezaa@covab.mak.ac.ug, \\ katodrago@yahoo.com, *sylvain.bieler@finddx.org, joseph.ndungu@finddx.org
}

How to cite this paper: Matovu, E., Edielu, A., Ojom, J., Nanteza, A., Kato, C.D., Biéler, S. and Ndung'u, J.M. (2019) Field Evaluation of LED Fluorescence Microscopy for Demonstration of Trypanosoma brucei rhodesiense in Patient Blood. Microscopy Research, 7, 1-9.

https://doi.org/10.4236/mr.2019.71001

Received: December 18, 2018

Accepted: January 28, 2019

Published: January 31, 2019

Copyright $\odot 2019$ by author(s) and Scientific Research Publishing Inc. This work is licensed under the Creative Commons Attribution International License (CC BY 4.0).

http://creativecommons.org/licenses/by/4.0/

\begin{abstract}
Diagnosis of Trypanosoma brucei rhodesiense human African trypanosomiasis requires demonstration of parasites in body fluids by microscopy. The microscopy methods that are routinely used are difficult to deploy in resource-limited settings due to practical challenges, including lengthy and tedious procedures, and the need for specific equipment to centrifuge samples in glass capillary tubes. We report here on a study that was conducted in a rural region of eastern Uganda to evaluate new methods that take advantage of a field-deployable LED fluorescence microscope. Examination of acridine orange-stained blood smears by LED fluorescence microscopy resulted in a diagnostic accuracy that was similar to that of routine methods, while the time needed to identify parasites was shortened significantly. These findings make these new microscopy methods attractive alternatives to procedures that are currently used for diagnosis of $T$. b. rhodesiense human African trypanosomiasis.
\end{abstract}

\section{Keywords}

Human African Trypanosomiasis, Rhodesiense,

LED Fluorescence Microscopy, Red Blood Cell Lysis, Diagnosis

\section{Introduction}

Human African trypanosomiasis (HAT, also known as sleeping sickness) manifests in two forms: as a chronic disease caused by Trypanosoma brucei gambi- 
ense that is prevalent in central and western Africa and an acute T. b. rhodesiense form in eastern and southern Africa [1]. These diseases are of importance in rural sub-Saharan Africa, although a progressive decrease in their incidence has put them in the spotlight for elimination as a public health problem [2]. Control of HAT largely relies on screening and treatment of infected individuals. For this, accurate diagnosis plays a central role, since case definition is based on demonstration of trypanosomes in body fluids, including blood, lymph node aspirates, and cerebrospinal fluid by microscopy [3]. In the absence of any serological test to screen for T. b. rhodesiense HAT, parasitological methods based on microscopy are solely relied upon to screen all individuals presenting with suggestive symptoms, in order to conclusively diagnose the disease, posing a huge burden to the strained healthcare system. As a result, most patients presenting with a fever are often tested for malaria, for which rapid diagnostic tests are available, and rarely for HAT, with the risk that many HAT cases can be missed. Current parasitological techniques are lengthy, elaborate and tedious, exerting undue strain on the usually understaffed rural health facilities where HAT is typically endemic. It is therefore pertinent that current microscopy methods be simplified in order to reduce the time taken to diagnose a single case.

A simple and rapid approach to reduce the laboratory workload and to increase the sensitivity of direct smear microscopy may now be possible through the introduction of a new fluorescence microscope based on the use of ultra-bright light emitting diodes (LED) developed jointly by Zeiss (Germany) and the Foundation for Innovative New Diagnostics (FIND), the Primo Star iLED [4]. By using an LED light source, Zeiss reduced the instrument's power consumption and increased the lifespan of the light source (up to 10,000 hours), making it much more affordable than classical fluorescence microscopes. The microscope can also be easily switched from bright-field light mode to fluorescence mode, and can be operated using a battery or solar power. For examination under fluorescent light, slides are stained with acridine orange (AO), while conventional light microscopy relies on Giemsa stain. AO staining has previously been shown to enhance parasite detection in the blood of HAT patients using the quantitative buffy coat (QBC) technique [5]. However, the use of QBC has been limited due to practical challenges in rural settings.

The performance of LED fluorescence microscopy methods to detect trypanosomes in thin or thick smears prepared with either whole or lysed blood was evaluated earlier using experimental infections at laboratories in the Democratic Republic of the Congo (DRC) and Uganda [6], and on T. b. gambiense HAT patients in the DRC [7]. However, the clinical performance of these new methods for the T. $b$. rhodesiense form of the disease has not been evaluated.

This study was carried out to compare the accuracy of LED fluorescence microscopy with routine parasitological methods in a district of eastern Uganda that is endemic for $T$. $b$. rhodesiense HAT. A previously described concentration method that differentially lyses red blood cells prior to preparation of smears [6] 
was included to determine whether visualisation of parasites would be improved, or the time to detect trypanosomes on a smear shortened.

\section{Materials and Methods}

\subsection{Study Sites and Ethical Considerations}

This study was carried out at Lwala mission hospital, Kaberamaido district, in the T. b. rhodesiense HAT endemic region of eastern Uganda. The hospital has been treating the highest number of $T$. $b$. rhodesiense HAT cases in the country. The study was carried out in conformity with the Declaration of Helsinki and guidelines for research involving human subjects outlined by the Uganda National Council for Science and Technology (UNCST). Ethical review was carried out by the Vector Control Division (Ministry of Health) followed by approval of the study by the UNCST.

\subsection{Study Design and Execution}

This was a prospective case-control study. All participants were enrolled after written informed consent in the presence of independent witnesses. Twelve $\mathrm{ml}$ of venous blood were collected into a heparinized Vacutainer from each participant and labelled with a blinding code by a nurse before handing over to two laboratory technicians for analysis. The first technician performed the haematocrit centrifugation technique (HCT [8], also known as capillary tube centrifugation), which was the most sensitive technique available on site, and prepared blood smears as described below. The second technician prepared and examined wet smears and performed the red blood cell (RBC) lysis and concentration procedure, as described below. Cases were defined as individuals with signs/symptoms suggestive of HAT, in whom trypanosomes were demonstrated by the HCT. A lumbar puncture was performed on all cases, and the cerebrospinal fluid (CSF) examined by microscopy to determine the stage of disease, a requirement to guide the treatment to be used [9]. All confirmed HAT cases were treated according to the national guidelines. Controls were defined as individuals presenting at the hospital with neither symptoms suggestive of HAT, nor history of HAT, and for whom the HCT was negative.

\subsection{Preparation of Microscopy Slides}

Microscopy slides were prepared by two technicians operating independently of each other, and uninformed of results obtained by the clinicians. The first technician prepared 6 thick smears using $5 \mu$ l blood for each, for subsequent staining as described below. The second technician prepared wet smears using $5 \mu$ blood, examined them immediately for a duration of 10 minutes, or until the first parasite was seen. For positive samples, the time taken to see the first trypanosome was recorded. Slides were scored as negative if no trypanosomes were detected in 10 minutes. The second technician also prepared three $15-\mathrm{ml}$ Falcon tubes per participant, each containing $3 \mathrm{ml}$ of blood; then treated them with $9 \mathrm{ml} \mathrm{RBC} \mathrm{ly-}$ 
sis solution (Qiagen) as previously described [6]. After the lysis procedure, the samples were centrifuged as described in [6], the resultant cell pellets each re-suspended in $40 \mu \mathrm{l}$ of supernatant, pooled, and 3 thick as well as 3 thin smears prepared using $20 \mu \mathrm{l}$ of the suspension. To further blind the samples, all slides were labelled with randomly generated numbers, from a coding list specifically prepared for the study. The slides were stained for 3 (AO) or 45 minutes (Giemsa) as previously described [6], rinsed and dried, and subsequently stored in opaque slide boxes.

\subsection{Reading of Stained Slides}

Four stained slides from each participant were examined under the microscope by one technician soon after processing: 1 thick blood smear stained with Giemsa, 1 thick blood smear stained with AO, 1 thin blood smear stained with $\mathrm{AO}$ after RBC lysis, and 1 thick blood smear stained with $\mathrm{AO}$ after RBC lysis. Stained slides were not examined by the technician who prepared the slides, but by the other technician. The time taken to detect the first trypanosome was recorded. Any slide in which no trypanosome was detected within 10 minutes was considered negative. The remaining slides were stored as back-up.

Slides stained with Giemsa were examined by bright field microscopy, while slides stained with AO were assessed by fluorescence microscopy. A Primo Star iLED microscope (Zeiss) was used for both bright field and fluorescence microscopy. Results were scored based on the number of parasites observed in 5 fields under a $400 \times$ magnification, as + for $1-4$ trypanosomes observed in a slide; ++ for 5 - 9 trypanosomes or +++ for 10 or more trypanosomes. After examination, the slides were stored in the slide box and kept in a cool, dry place, for monitoring and independent assessment.

\subsection{Statistical Analysis}

Data analysis was done using IBM SPSS version 22 statistical software. Deviation from normality was tested using D'Agostino-Pearson normality test. Numerical variables were summarized using mean and standard error of the mean. Comparison between the different methods of slide preparation was done using a one-way ANOVA followed by Tukey's multiple comparison test, set at a significance level of $5 \%$.

\section{Results}

Sixty eight participants (41 cases and 27 controls) were included in the study between April and September 2011 (35 participants), and between December 2012 and June 2013 (33 participants). The male:female ratio for the cases was 0.28 , with a mean age of $28.2 \pm 3.1$ years (Table 1 ). The sensitivity of all the parasitological methods performed in the study was $100 \%$, with all the 41 cases correctly diagnosed as positive. A large number of cases $(32,78.0 \%)$ were classified as late stage, with trypanosomes detected in the CSF of $29(70.3 \%)$ of them. 
The mean CSF white blood cell (WBC) count was $46.1 \pm 7$ cells/ $\mu$ l (range 3 $158)$. When the number of trypanosomes per field in the blood was compared across the different methods, a higher parasite density ( $\geq 10$ trypanosomes/field) was detectable in thin smears stained with AO after lysis and concentration (11, $29.7 \%)$ and in HCT $(10,23.4 \%)$ (Figure 1). High parasite densities were detectable at lower frequencies in wet smears $(3,7.3 \%)$ and in thick, AO stained smears $(2,4.9 \%)$. All 27 controls were negative by all the microscopy and staining methods investigated, indicating no false positivity.

The average time taken to see the first trypanosome on a slide was significantly influenced by the method of preparation $\left(\mathrm{F}_{(4,200)}=3.64, \mathrm{p}<0.007\right)$. When average time taken to read a slide was compared across preparation methods, a significantly $(\mathrm{p}<0.05)$ shorter time was recorded for thin smears stained with AO after lysis and concentration $(190.9 \pm 18.3 \mathrm{sec})$ as compared to all the other methods (Figure 2). When thin and thick smears stained with AO after lysis and concentration were compared, time to detection of parasites in AO stained thick smears was longer (on average $241.6 \pm 20.1 \mathrm{sec}$ ). It took longest to detect trypanosomes from Giemsa stained thick smears $(294.2 \pm 21.1 \mathrm{sec})$. Interestingly, there was no significant difference $(p>0.05)$ in the time taken to detect trypanosomes in thick AO stained smears of whole blood $(273.1 \pm 21.7 \mathrm{sec})$ as compared to wet smears $(269 \pm 23.1 \mathrm{sec})$. When the average time taken to see the first trypanosome on a slide was correlated with parasite density, there was an inverse relationship that was strongest with thin smears stained with AO after lysis and concentration (Pearson correlation $-0.745, \mathrm{p}<0.0001$ ).

Table 1. Characteristics of T. b. rhodesiense HAT cases.

\begin{tabular}{ccccc}
\hline Characteristic & Stage 1 & Stage 2 & Total & p value \\
\hline Age (mean \pm SEM) & $23.8 \pm 5.9$ & $29.4 \pm 3.7$ & $28.2 \pm 3.1$ & 0.465 \\
Sex & & & & \\
Male & $7(21.9 \%)$ & $25(78.1 \%)$ & $32(78 \%)$ & 0.65 \\
Female & $2(22.2 \%)$ & $7(77.8 \%)$ & $9(22 \%)$ & \\
CSF WBC count (mean \pm SEM) & $4.1 \pm 0.5$ & $57.9 \pm 7.8$ & $46.1 \pm 7.0$ & $0.0001^{*}$ \\
$\begin{array}{c}\text { Trypanosomes in CSF } \\
\text { Parasitaemia by HCT } \\
\text { (tryps } \text { capillary) }\end{array}$ & 0 & $29(90.6 \%)$ & $29(70.3 \%)$ & $0.0001^{\star}$ \\
1 - 4 & & & & \\
$5-9$ & $2(25 \%)$ & $6(75 \%)$ & $8(19.5 \%)$ & 0.575 \\
$\geq 10$ & $6(26.1 \%)$ & $17(73.9)$ & $23(56.1 \%)$ & \\
& $1(10 \%)$ & $9(90 \%)$ & $10(24.4 \%)$ &
\end{tabular}

* indicates significant differences between disease stages. SEM: standard error of the mean; CSF: cerebrospinal fluid; WBC: white blood cell; HCT: haematocrit centrifugation technique; tryp: trypanosome. 


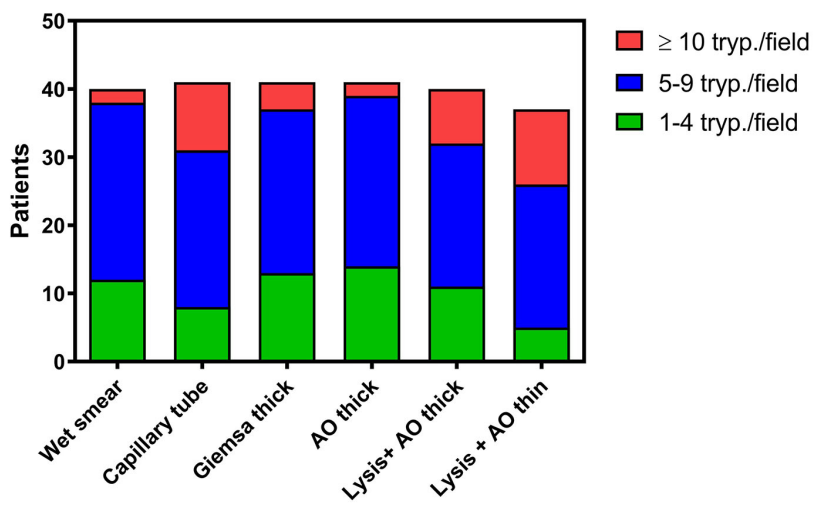

Figure 1. Parasite density compared across different microscopy methods. AO: acridine orange; tryp.: trypanosome. The total height of each bar does not always correspond to 41 patients, as some cases could not be tested with all the methods. For HCT, the parasites recorded were those observed per capillary tube, rather than per field as has been presented for all techniques for simplicity.

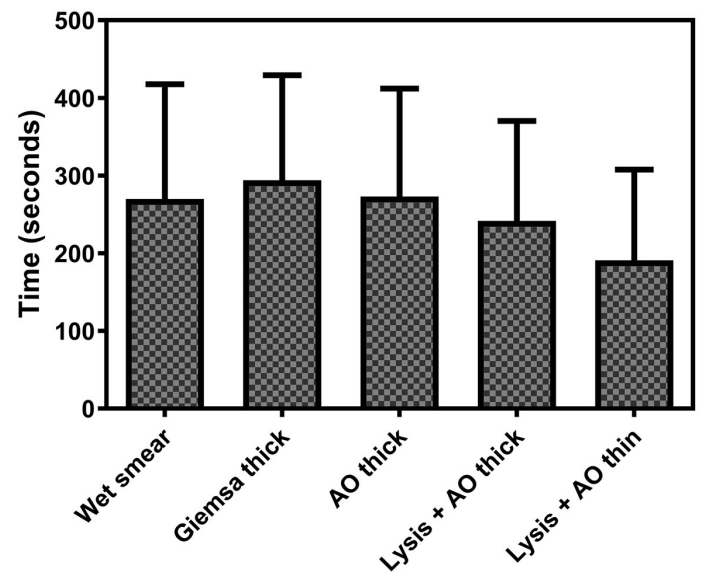

Figure 2. Average time taken to see the first trypanosome on a slide across different slide preparation methods. Bars indicate standard error of the mean; AO: acridine orange.

\section{Discussion}

Diagnosis of $T$. b. rhodesiense HAT is complicated by the absence of a screening test. There is therefore a justifiable need to simplify and improve methods for trypanosome detection. While all the smear microscopy methods that were evaluated here performed equally in terms of diagnostic sensitivity and specificity, the analytical sensitivity was highest with thin smears stained with AO after lysis and concentration, which is consistent with results obtained using experimental infections [6]. This is also consistent with the finding that this method was the most sensitive smear microscopy method to diagnose T. b. gambiense patients [7], who present a better dynamic range to detect subtle sensitivity differences, since they exhibit lower densities of parasites in the blood than T. $b$. rhodesiense patients [9]. Similarly, the observation that thin smears stained with 
$\mathrm{AO}$ after lysis and concentration was associated with the shortest time to detect parasites was also reported using experimental infections [6]. AO is also more attractive than Giemsa due to a faster procedure for staining slides; while Giemsa staining usually requires incubation for 20 to 50 minutes, $\mathrm{AO}$ staining can be achieved in only 3 minutes.

Although in the present study LED fluorescence microscopy was not found to provide any advantage over HCT in terms of diagnostic accuracy, it could facilitate access to parasitological testing in peripheral sites, where equipment to centrifuge glass capillary tubes is often missing. In addition, unlike with methods relying on fresh samples such as HCT, samples prepared onto slides can be stored and sent to reference laboratories. Indeed, we have piloted a referral system in which a few cases, who would not have accessed Lwala mission hospital, have been detected by checking smears sent to the hospital (unpublished data). Another advantage would be in a situation when the laboratory staff cannot immediately examine fresh blood to detect live trypanosomes, as we found that examining stained smears was as sensitive as other methods using fresh samples.

Since this study was completed, the LED fluorescence microscopy methods that were evaluated here have been introduced in five health facilities located around a conservation area in northern Malawi, where they have been used to enhance diagnosis of $T$. $b$. rhodesiense HAT, in combination with other routine methods. In addition, these methods have been introduced in multiple facilities in Uganda [10], Guinea, Chad, the Democratic Republic of the Congo, the Republic of the Congo, Angola, South Sudan and Nigeria, where they have contributed to improved diagnosis of $T$. b. gambiense HAT.

Finally, considering the current low prevalence of $T$. $b$. rhodesiense HAT and trend toward integration of disease control activities with general health services, the microscopy methods evaluated in the present study may also provide useful options for diagnosing other infections. In particular, LED fluorescence microscopy has been shown to be more sensitive than conventional Ziehl-Neelsen microscopy and has been recommended by the World Health Organization for diagnosis of tuberculosis [11]. LED fluorescence microscopy using acridine orange has also been shown to be an accurate and fast method to diagnose Plasmodium falciparum infections [12]. Other parasites have been reported to be easily visualized by fluorescence microscopy after staining with acridine orange, such as Trichomonas vaginalis and Leishmania donovani [13] [14]. Thus, the utility of LED fluorescence microscopy in the diagnosis of other infections would deserve to be investigated further in order to determine how these novel tools could be best used to integrate diagnosis of multiple diseases in resource-limited settings.

\section{Conclusion}

While examination of acridine orange-stained blood smears by LED fluorescence microscopy did not result in any observable improvement in accuracy in comparison to the routine microscopy methods used to diagnose $T$. b. rho- 
desiense HAT, there was a significant reduction in the time needed to identify parasites when using these new methods. These methods could therefore be considered as alternatives to current diagnostic procedures for $T$. b. rhodesiense $\mathrm{HAT}$, as they would improve throughput and free some time for the technicians to perform other routine tasks in their typically high-workload laboratories. Since acridine orange is known to stain various parasites, the fluorescence microscopy methods that were evaluated here could also be of interest for use in diagnosis of other parasitic diseases.

\section{Acknowledgements}

We wish to thank clinical and technical staff at Lwala mission hospital for assistance with enrolment of participants, preparation and examination of slides. This study was funded by the Bill \& Melinda Gates Foundation and the Department for International Development of the United Kingdom through FIND.

\section{Conflicts of Interest}

The authors declare no conflicts of interest regarding the publication of this paper.

\section{References}

[1] World Health Organization (1998) Control and Surveillance of African Trypanosomiasis. World Health Organization Technical Report Series 881.

[2] Franco, J.R., Cecchi, G., Priotto, G., Paone, M., Diarra, A., Grout, L., Mattioli, R.C. and Argaw, D. (2017) Monitoring the Elimination of Human African Trypanosomiasis: Update to 2014. PLoS Neglected Tropical Diseases, 11, e0005585. https://doi.org/10.1371/journal.pntd.0005585

[3] World Health Organization (2013) Control and Surveillance of Human African Trypanosomiasis: Report of a WHO Expert Committee. World Health Organization Technical Report Series 984.

[4] ZEISS Primo Star iLED—The Flexible, Complete Solution for Testing Tuberculosis. https://www.zeiss.com/microscopy/int/products/light-microscopes/primo-star-iled. $\underline{\mathrm{html}}$

[5] Bailey, J.W. and Smith, D.H. (1992) The Use of the Acridine Orange QBC Technique in the Diagnosis of African Trypanosomiasis. Transactions of the Royal Society of Tropical Medicine and Hygiene, 86, 630. https://doi.org/10.1016/0035-9203(92)90160-E

[6] Biéler, S., Matovu, E., Mitashi, P., Ssewannyana, E., Karhemere, S., Bessell, P.R. and Ndung'u, J.M. (2012) Improved Detection of Trypanosoma brucei by Lysis of Red Blood Cells, Concentration and LED Fluorescence Microscopy. Acta Tropica, 121, 135-140. https://doi.org/10.1016/j.actatropica.2011.10.016

[7] Mitashi, P., Lutumba, P., Lumbala, C., Bessell P., Bieler, S. and Ndung'u, J.M. (2015) Improved Detection of Sleeping Sickness Cases by LED Fluorescence Microscopy: Evidence from a Prospective Multi-Centric Study in the Democratic Republic of the Congo. Microscopy Research, 3, 17-25. https://doi.org/10.4236/mr.2015.32003

[8] Woo, P.T. (1970) The Haematocrit Centrifuge Technique for the Diagnosis of African Trypanosomiasis. Acta Tropica, 27, 384-386. 
[9] Brun, R., Blum, J., Chappuis, F. and Burri, C. (2010) Human African Trypanosomiasis. The Lancet, 375, 148-159. https://doi.org/10.1016/S0140-6736(09)60829-1

[10] Wamboga, C., Matovu, E., Bessell, P.R., Picado, A., Biéler, S. and Ndung'u, J.M. (2017) Enhanced Passive Screening and Diagnosis for Gambiense Human African Trypanosomiasis in North-Western Uganda-Moving towards Elimination. PLoS ONE, 12, e0186429. https://doi.org/10.1371/journal.pone.0186429

[11] World Health Organization (2011) Fluorescent Light-Emitting Diode (LED) Microscopy for Diagnosis of Tuberculosis: Policy Statement.

[12] Lenz, D., Kremsner, P.G., Lell, B., Biallas, B., Boettcher, M., Mordmüller, B. and Adegnika, A.A. (2011) Assessment of LED Fluorescence Microscopy for the Diagnosis of Plasmodium falciparum Infections in Gabon. Malaria Journal, 10, 194. https://doi.org/10.1186/1475-2875-10-194

[13] Mason, P.R., Super, H. and Fripp, P.J. (1976) Comparison of Four Techniques for the Routine Diagnosis of Trichomonas vaginalis Infection. Journal of Clinical Pathology, 29, 154-157. https://doi.org/10.1136/jcp.29.2.154

[14] Ndung'u, J.M., Bieler, S. and Roscigno, G. (2010) “Piggy-backing” on Diagnostic Platforms Brings Hope to Neglected Diseases: The Case of Sleeping Sickness. PLoS Neglected Tropical Diseases, 4, e715. https://doi.org/10.1371/journal.pntd.0000715 